\title{
La administración del Rorschach: La complejidad e integralidad del método*
}

Lupe Jara

Universidad de Lima

Recibido: 1 de julio del 2011 / Aprobado: 25 de julio del 2011

A partir de la revisión de 88 administraciones del Rorschach en estudiantes universitarios que se inician en el conocimiento y la capacitación de la prueba se busca conocer cómo son incorporadas y ejecutadas las diferentes instrucciones, consignas y pautas para su aplicación. Para ello, esta administración es registrada en una filmación con el consentimiento del examinado. Asimismo, mientras un alumno aplica la prueba, otro observa en la cámara Gesell, registrando cómo se realiza la administración. Los resultados indican dificultades en el establecimiento del rapport y en el manejo técnico de la prueba, en particular respecto a la encuesta. Se discuten las implicancias de estas dificultades vinculándolas con el desarrollo de las competencias de los estudiantes y con el impacto en el desempeño del examinado.

administración del Rorschach / entrenamiento de los evaluadores / rapport

The administration of the Rorschach test: Complexity and integrity of the method

The purpose of this study is to investigate how university students that are initiating their training in the knowledge of the administration of the Rorschach test incorporate and execute the instructions and procedures of its application. To this end, the administration of the test is filmed with the examined students' consent and while the students proceed with the test administration, another student registers the process utilizing a Gesell camera. Results show the difficulties establishing a good rapport and the technical handling of the test, particularly in regards to the survey. This study reveals the difficulties in the development of competencies and performance of the examined students.

administration of the Rorschach test / training of evaluators / rapport

\footnotetext{
* La autora agradece a la profesora Sylvia Rivera por compartir los videos realizados por sus alumnos en el curso dedicado al psicodiagnóstico de Rorschach, así como a Francesca Ruggiero y a Sophia Escurra, asistentes en este estudio.

Correo electrónico: Ljara@ulima.edu.pe
} 
En 1921, Hermann Rorschach creó la famosa prueba que lleva su nombre. Desde entonces este instrumento ha sido uno de los más usados e investigados en el mundo (Exner, 1994), lo que también ocurre en nuestro país (Ráez, 2006). Pero como señala Exner, no es hasta 1968, con la creación de la Fundación para la Investigación del Rorschach (Rorschach Research Foundation), que se inicia un largo proceso para la estandarización de las hasta entonces diversas formas de administrar, codificar e interpretar la prueba, lo que se consolida en 1974 con el nacimiento del Sistema Comprehensivo del Rorschach (SCR). Este, con el fin de satisfacer las condiciones psicométricas de validez y confiabilidad requeridas por una prueba psicológica, sustenta sus códigos y procedimientos en una base empírica sólida (Weiner, 1997).

De este modo, en la actualidad el SCR es considerado como uno de los métodos más completos, útiles y valiosos para evaluar la organización y funcionamiento de las personas, lo que le ha permitido consolidarse y expandirse a nivel mundial (Exner, 1994, 2005). Es justamente esta presencia internacional lo que lleva a varios autores (Allen \& Dana, 2004; Dana, 2009; Daroglou, 2009; Ephraim, 1996; Franchi \& Andronikof-Sanglade, 1999) a cuestionar si su aplicación, codificación e interpretación tienen un carácter universal; discutiendo la necesidad de incorporar la variable cultural en todo este proceso de evaluación.
En particular, la administración del Rorschach es un aspecto crucial y determinante en este proceso, pues como señalan Exner y Weiner (1995), la interpretación de los resultados requiere que la data sea recogida apropiadamente. Por su parte, Ephraim (1996) agrega que la interpretación integra varias fuentes de información, entre ellas las actitudes durante la administración (conducta tanto del evaluador como del examinado). De ahí que los primeros esfuerzos de la Fundación para la investigación del Rorschach estuvieran dirigidos hacia cuestiones básicas como la posición del examinado respecto al evaluador, la consigna, y el registro y encuesta de las respuestas (Exner, 1994).

El método del psicodiagnóstico de Rorschach comienza en el encuentro con el examinado. Como plantea Lerner (1996a, 1996b), esta situación tiene lugar en el contexto de una relación interpersonal que, por sus componentes intersubjetivos y experienciales, debe basarse en la empatía. A la vez, para Acklin (1994), el contexto alrededor de la evaluación convoca esquemas del examinado y del evaluador con el fin de adaptarse a la situación. Estos esquemas dirigen el modo de conducirse con la prueba o con el otro (evaluador o examinado) y generan ciertas expectativas o temores en sus participantes.

Así, por ejemplo, considerando el tipo de evaluación, la aplicación del Rorschach para un puesto de trabajo tiene un matiz distinto al de una evalua- 
ción clínica. En el caso del postulante al trabajo, este puede poner en juego sus esquemas de lo que significa una evaluación con respuestas correctas e incorrectas y puede tener dificultades para adaptarse a estímulos ambiguos, donde no tiene elementos para saber si está respondiendo "bien". Al respecto, Exner (1994) encuentra que los examinados preocupados por presentar un perfil aceptable, tienden a descartar varias de las respuestas que habían elaborado por considerarlas no adecuadas a la situación de evaluación. Lerner (1996a) agrega que el desempeño del individuo involucra lo que este cree que debe hacer con la tarea que se le ha asignado.

Si nos enfocamos en el grupo examinado, también será distinto aplicar el Rorschach a un grupo de estudiantes universitarios (examinados constantemente de diferentes maneras) que a un grupo de mujeres promotoras de comedores populares (que quizás no cuenten con la experiencia de haber sido evaluadas). Obviamente, los primeros al disponer de una mayor provisión de esquemas respecto a una situación de evaluación, tienen mayores posibilidades de ajustarse a ella. Una manera de investigar este aspecto es a través de la comprensión de las consignas, el número de respuestas, la presencia de shocks -no responder ante alguna(s) lámina(s) $o$ ante el test-, etcétera.

En el caso del evaluador, si este es principiante, puede carecer de esquemas previos que le permitan resolver al- gunas dificultades que se presenten en la evaluación. O si, tal como lo sugiere Lerner (1996b), el evaluador considera la situación de evaluación como un encuentro interpersonal, donde debe poner en juego su empatía, probablemente obtenga resultados distintos a cuando asimila la evaluación como un medio rápido para seleccionar personal.

Al respecto, Exner (1994), basándose en una serie de experimentos, señala que los evaluadores más expertos y más cálidos en su trato obtienen un número mayor de respuestas humanas y más protocolos de longitud media; mientras que la incomodidad del evaluador produce ansiedad en el examinado y le hace comportarse de manera más reservada. A la vez, Lis, Parolin, Calvo, Zennaro \& Meyer (2007) también encuentran que los examinados que se muestran desconfiados y poco cooperativos tienden a dar protocolos simples. Lo contrario es igualmente cierto, quienes se sienten más cómodos y tienen una mayor apertura, brindan protocolos más largos y complejos. Así, los pacientes que son examinados por su propio terapeuta producen protocolos más complejos y reveladores que aquellos que son examinados por un terapeuta diferente.

De este modo vemos que si bien el SCR prescribe un conjunto invariante de instrucciones para la administración del Rorschach, se conforma un nuevo esquema cada vez. Por ello es importante identificar las características del 
esquema puesto en juego durante la evaluación, explorar las expectativas o temores del examinado y clarificar los aspectos del encuadre y la consigna. Al respecto, Hartmann (2001, en Lis et al., 2007) encuentra que realizar una introducción corta genera que se le hagan más preguntas al evaluador sobre la prueba y que el protocolo sea corto.

Por otro lado, la administración del Rorschach se complica para el evaluador en la fase de la encuesta. Si bien su objetivo es bastante evidente-clarificar la respuesta tal como fue emitida con el fin de que la codificación se haga con la mayor precisión posible-, el procedimiento no es tan fácil de conducir, por lo que es uno de los elementos del SCR peor comprendidos y utilizados (Exner, 1994, 2003). Por ejemplo, aunque el SCR establece que las preguntas en la encuesta deben hacerse de manera indirecta (Exner, 2003), Lis et al. (2007) reportan el uso de preguntas directas, las cuales incrementan los puntajes de color, movimiento y sombreado.

A la vez, Lis et al. (2007) encuentran que el entrenamiento y la experiencia con la encuesta y la codificación pueden tener un gran efecto en los puntajes del SCR. Así los sujetos examinados por evaluadores expertos lucen más complejos, articulados y comprometidos con la tarea que los examinados por evaluadores con un menor entrenamiento y experiencia; sin embargo, los primeros también presentan una mayor percepción inusual y lapsus en su lógi- ca. De esta manera, una explicación al Lambda alto (protocolo más simple) en muestras diferentes a las de Exner podría deberse antes que a un factor cultural a la realización de una encuesta insuficiente.

Como se aprecia, el efecto evaluador impacta de manera importante en los puntajes del SCR, lo que puede modificar significativamente la interpretación del protocolo. Al respecto, Vinet (2000) encuentra que el Lambda alto (defensividad), bajo Afr y C (bajo involucramiento afectivo) e $\mathrm{Y}$ (ansiedad) se presentan en países latinoamericanos donde existen fuertes diferencias entre el evaluador y el examinado, lo que da lugar a comportamientos enmascarados de ansiedad interiorizada, pues el examinado se encuentra muy atento a las necesidades del evaluador, tratando de evitar una situación difícil. Como señala Daroglou (2009), es necesario considerar que el procedimiento de administración del Rorschach puede ser una experiencia nueva para el examinado y no parte de su panorama cultural.

Por otro lado, al plantear el uso transcultural del Rorschach, Dana (2009) y Daroglou (2009) llaman la atención sobre el entrenamiento de los evaluadores, quienes siguen dependiendo de aspectos procedimentales que les resultan disímiles a ellos mismos, a la par que analizan e interpretan la data usando teorías desarrolladas en sociedades occidentales. Para Dana es necesario que los evaluadores modifiquen 
sus expectativas sobre las conductas y el performance del examinado frente al Rorschach a partir de incorporar la perspectiva cultural local, no proveída por la academia. Así, lograr competencia en la investigación multicultural requiere reexaminar la credibilidad y las limitaciones del entrenamiento profesional para nuevas poblaciones del interés del investigador, pero también demanda un enorme investimiento en nuevos aprendizajes sobre la vida, los valores, las experiencias y la visión del mundo de otros, quienes son marcadamente diferentes al investigador.

Para Dana (2009) incorporar la perspectiva cultural al SCR abarca los procedimientos de administración y reporte del protocolo, de codificación de las respuestas y de generar inferencias consensuales sobre los puntajes. Ello, reconociendo que las transformaciones fiables son más difíciles. Así, en la incorporación de esta perspectiva cultural, Dana recomienda analizar el efecto de los factores culturales en la evaluación, tomando en cuenta también el estatus de la aculturación. De esta manera, desarrollar las competencias necesarias para la comprensión de la cultura del examinado puede incluir habilidades del lenguaje tanto como conocimiento experiencial y cognitivo, a la par que familiarizarse con las convenciones culturales específicas para establecer un adecuado rapport. Lo anterior implica combinar conductas y afectos de un modo aceptable y confortable para el examinado. Tomar en cuenta estos aspectos antes de iniciar el contacto con el examinado permite sugerir la naturaleza de la preparación requerida para conducir la administración e interpretación de la data del Rorschach.

A partir de lo revisado se aprecia la importancia de una adecuada administración del Rorschach, por lo que este estudio busca conocer cómo son incorporadas y ejecutadas las diferentes instrucciones, consignas y pautas para la administración del Rorschach en un grupo de estudiantes universitarios que se inician en el conocimiento y la capacitación de esta prueba.

\section{MÉTOdo}

\section{Unidad de estudio}

Se revisan 88 administraciones del psicodiagnóstico de Rorschach realizadas por estudiantes universitarios que se inician en el conocimiento y la capacitación de esta prueba, mediante un curso ubicado en el octavo nivel de la malla curricular de la carrera de Psicología en una universidad privada de Lima metropolitana.

La administración del psicodiagnóstico de Rorschach es registrada en una filmación con el consentimiento de la persona examinada. En la administración participan dos estudiantes: uno aplicando la prueba y el otro, ubicado en la Cámara Gesell, registrando cómo se realiza esta aplicación. Entre los estu- 
diantes que aplican la prueba el 80,2\% son mujeres y el $19,8 \%$ son hombres.

\section{Técnica de recolección de} información

El sistema comprehensivo del Rorschach prescribe un conjunto invariante de instrucciones para la administración de la prueba. A partir de estas instrucciones se ha construido un registro de observación para verificar si estas se cumplen durante la aplicación del Rorschach. Este registro abarca las siguientes áreas: condiciones ambientales, posición evaluador-examinado, preparación de los materiales, establecimiento del rapport con el examinado, seguimiento de las consignas, procedimiento de aplicación en la fase de respuesta, procedimiento de aplicación en la fase de encuesta y cierre de la aplicación. A la vez, se recoge la autoevaluación del evaluador sobre su desempeño durante la administración del Rorschach.

\section{Procedimiento}

Inicialmente, se capacita a los estudiantes en la administración del Rorschach de manera teórica y práctica. En el campo teórico se detalla el conjunto de instrucciones establecidas para su administración, fundamentándolas en los estudios empíricos realizados por la Fundación para la Investigación del Rorschach. A la vez, en el nivel práctico se muestran, a través del modelamiento, el role playing y la visualización de videos, tanto el procedimiento correcto como los errores más frecuentes y las situaciones difíciles que pueden presentarse en la aplicación de la prueba. Posteriormente, los estudiantes forman díadas y contactan con un examinado voluntario para realizar la aplicación, la cual es filmada bajo su consentimiento informado. Mientras uno de los estudiantes administra la prueba, el otro, ubicado en la Cámara Gesell, observa y registra cómo se hace esta administración. Al término de la aplicación los estudiantes elaboran el informe de la administración en la que incluyen tanto el registro de observación como la autoevaluación del evaluador. Para el análisis de la administración, en un primer momento se contrastó el registro de observación con la filmación, encontrándose que el estudiante observador tendía a ignorar las dificultades o errores presentes en la administración, optándose por efectuar un nuevo registro de la filmación, a cargo de dos asistentes entrenados en la administración de la prueba. A partir de este nuevo registro se desarrolla el análisis descriptivo de la información recogida.

\section{RESUltados}

Como toda prueba psicológica, el Rorschach debe administrarse en un ambiente cómodo y libre de ruidos e interrupciones. A la vez, dada su naturaleza -prueba conformada por estímulos visuales- se hace necesario contar con luz adecuada, de preferencia luz del día. Al 
respecto, todas las administraciones se llevan a cabo durante el día o utilizando luz blanca, lo que brinda una mayor nitidez al sujeto para examinar las láminas. Asimismo, la gran mayoría realiza la aplicación libre de ruidos e interrupciones, disponiendo los muebles en búsqueda de comodidad. Dado que estas condiciones estarían garantizadas en un consultorio provisto por la universidad, llama la atención el porcentaje de pruebas que se aplican con interrupciones, como las causadas por los sonidos de los teléfonos celulares (10\%); los ruidos provenientes de ambientes aledaños (13\%), o la mala disposición de los muebles (19\%). En particular, son los evaluadores hombres (29\% versus $16 \%$ de las mujeres) quienes menos atención prestan a la disposición de los muebles para que tanto ellos como los examinados se encuentren cómodos.

Otro aspecto crucial en la administración del Rorschach es la posición del evaluador con respecto al examinado, la cual "nunca debe ser cara a cara" (Exner, 2003, p. 16). Esta posición solo la utiliza un evaluador varón, mientras la posición lateral (examinado y evaluador, uno al lado del otro), recomendada por el SCR, es seguida por el $93 \%$ de los evaluadores. Adicionalmente, la autora ha planteado que cuando el evaluador sea diestro, ubique al examinado hacia su izquierda y cuando sea zurdo lo haga a su derecha con el fin de que el brazo con el que escribe no interfiera en la proximidad corporal entre ellos ni dificulte la toma de notas. Al respecto, el $71 \%$ de los evaluadores diestros sigue esta indicación, sobre todo los hombres ( $80 \%$ versus el $68 \%$ en el caso de las mujeres). Mientras en el caso de los evaluadores zurdos, el único varón sigue esta instrucción al igual que tres de las cinco mujeres. Finalmente, la posición diagonal, alternativa a la posición lateral, solo la realizan cinco mujeres.

Asimismo, el evaluador requiere preparar sus materiales. Sobre este punto todos los estudiantes disponen de hojas suficientes para realizar sus anotaciones y la gran mayoría (94\%) ha ordenado las láminas consecutivamente con la lámina I al inicio, a la vez que las ha apilado por el reverso (89\%), aunque el $25 \%$ de los varones (versus el $8 \%$ de las mujeres) no tiene las láminas apiladas por el reverso. Del mismo modo, las láminas deben estar a la vista del examinado, pero no a su alcance; indicación seguida por el $41 \%$ de los evaluadores (el 21\% no coloca las láminas a la vista del examinado y el $38 \%$ las coloca a su alcance). Por último, la hoja de localización no debería estar a la vista del examinado, instrucción realizada por el $82 \%$ de los evaluadores.

Antes de la aplicación de las láminas es necesario establecer el rapport con el examinado, como sucede en cualquier evaluación psicológica, pero dada la inestructuración de esta prueba -que puede despertar una mayor incertidumbre y ansiedad-, ello es más indispensable con el Rorschach. Al respecto, 
encontramos que la casi totalidad de los evaluadores (95\%) no le dedica un tiempo suficiente al establecimiento del rapport (menos de 6 minutos), incluso la mitad no le dedica ningún tiempo, pasando del saludo al examinado a la administración de las láminas. Entre los que dedican unos pocos minutos al rapport, el grueso de los evaluadores no explica el procedimiento de la administración (45\%), ni registra los datos básicos (datos de filiación) (52\%) o antecedentes (73\%) del examinado. En particular, son las mujeres quienes menos consultan los datos de filiación (54\% versus $41 \%$ en el caso de los varones) o algunas referencias del examinado ( $76 \%$ versus $59 \%$ en el caso de los varones). Como consecuencia, la gran mayoría (84\%) concluye la aplicación en menos de 40 minutos (siendo lo usual utilizar entre 45 minutos a una hora), lo que sucede sobre todo con las mujeres ( $87 \%$ versus el $75 \%$ de los hombres).

Esta premura a lo largo de la aplicación lleva a que si bien la mayor parte de los evaluadores luce calmada (57\%), un grueso de ellos se muestra apurado (40\%). En contraste, los examinados se presentan calmados (78\%), siendo menor el grupo que luce apurado (15\%) o ansioso (7\%). En particular, los examinados lucen apurados ante el evaluador hombre (24\% versus el $13 \%$ en la evaluadora mujer) y ansiosos ante la evaluadora mujer (cinco versus uno frente al evaluador hombre). Lo anterior da lugar a que en la interacción evaluador- examinado si bien prima la cordialidad $(52 \%)$, existe un porcentaje elevado (47\%) en el que la interacción es distante. Pese a ello, ningún examinado presenta una situación de bloqueo ante alguna de las láminas (no obstante ser el bloqueo una señal de ansiedad ante la prueba); aunque ante la falta de rapport, el 52\% realiza preguntas al evaluador durante la aplicación de la prueba. ${ }^{1} \mathrm{Fi}$ nalmente, al término de la aplicación, la mayor parte de los evaluadores (85\%) no se despide del examinado de manera cordial y apropiada, cerrando la evaluación con prisa.

En tanto, durante la administración misma del Rorschach, solo el 35\% de los evaluadores presenta la prueba. Sin embargo, el $87 \%$ cumple con utilizar la consigna correcta ("¿Qué podría ser esto?"). Asimismo, todos entregan las láminas en la posición correcta y la gran mayoría (83\%) las entrega en las manos del examinado; aunque el $29 \%$ de los hombres no lo hacen con la lámina I, mejorando para las siguientes láminas $(12,5 \%$ sigue sin corregir esta indicación). Por su parte, el $73 \%$ de los examinados toma las láminas en sus manos.

1 Entre las preguntas que realiza el examinado se encuentran: “¿Lo primero que me venga a la mente, no?”, “¿puedo voltear la lámina?”, “¿tengo que responder a toda la mancha o a partes?", “¿puedo decir varias cosas?”, “¿puedo decir cualquier cosa?”, “„no importa si suena incoherente lo que veo?”, “cómo se llama...?”, entre otras. 
Entre las situaciones problemáticas que pueden presentarse durante la aplicación, se encuentra que en la primera lámina el examinado brinde una única respuesta (situación que de mantenerse en el resto de láminas generaría un protocolo con menos de 14 respuestas, haciéndolo inválido), frente a lo cual el evaluador debe intervenir indicando al examinado que puede dar más respuestas. Esta situación se presenta en el 29\% de los casos, siguiendo la instrucción correspondiente todos los evaluadores hombres y el $70 \%$ de las mujeres. De otro lado, cuando el examinado brinda cinco respuestas y tiene la intención de seguir dando más respuestas, el evaluador debe intervenir sugiriendo pasar a la siguiente lámina. Lo anterior ocurre en el $11 \%$ de los casos, siguiendo la indicación el 20\% de los evaluadores.

Al término de la primera fase de respuesta, el evaluador vuelve a pasar las láminas para realizar la encuesta. Para ello, requiere reordenar las láminas, con la lámina I encima, acción que realiza el $62 \%$ de los evaluadores. A su vez, el evaluador debe explicar los objetivos de la encuesta, los cuales son clarificar la localización y los determinantes. Esta explicación es efectuada por el $61 \%$ de los evaluadores mientras el $15 \%$ no la realiza, el $15 \%$ solo explica la localización y el $10 \%$ solo los determinantes.

Luego, al comenzar la encuesta, el evaluador debe repetir textualmente toda la respuesta del examinado, lo que es realizado por el $84 \%$ de los evaluado- res, entre los que destacan las mujeres ( $88 \%$ versus $69 \%$ de los hombres). Las respuestas además deben ser encuestadas de manera individualizada, acción llevada a cabo por todos los evaluadores. Asimismo, se debe encuestar la localización y los determinantes de cada respuesta. Al respecto, la localización es encuestada en el $77 \%$ de los casos, especialmente por los hombres (94\% versus $72 \%$ por las mujeres); mientras que existen más fallas en la encuesta de los determinantes. Así, el $46 \%$ no encuesta los determinantes, el $25 \%$ lo hace solo al inicio, en particular los hombres (44\% versus $20 \%$ las mujeres), el $20 \%$ lo hace la mayor parte de las veces y solo el $10 \%$ lo hace con todas las respuestas, siendo todas ellas mujeres.

El SCR especifica qué tipo de preguntas pueden realizarse o no en la encuesta. Sobre este punto, encontramos que el $94 \%$ no encuesta aspectos relevantes de las respuestas, mientras el $21 \%$ hace preguntas innecesarias. A la vez, la pregunta más frecuente $(35 \%)$ utilizada para elicitar los determinantes es "¿Qué te hizo pensar en (respuesta)?". De otro lado, si bien durante la fase de respuesta casi todos los evaluadores $(97 \%)$ copian literal y completamente todo lo dicho por el examinado, no ocurre así en la encuesta, en la que este porcentaje baja a $68 \%$.

Adicionalmente, se registraron una serie de errores no previstos en la ficha de observación que se presentan en el siguiente cuadro: 
Rapport: El evaluador toma los datos de filiación y antecedentes al concluir la aplicación, el evaluador trata de "usted" a un examinado joven marcando un trato distante, el evaluador se centra en sus apuntes y no mira al sujeto.

Fase de respuesta: El evaluador estimula por más respuestas al sujeto en la primera lámina cuando el sujeto ya ha dado espontáneamente más de una respuesta, asimismo estimula por más respuestas en otras láminas.

Manejo de las láminas: El evaluador ubica las láminas en sus piernas, las láminas se encuentran apiladas y ordenadas boca arriba, las láminas por momentos están a la vista del examinado y por otros momentos no, las láminas están mal ordenadas, presenta las láminas en desorden en la fase de respuesta y de encuesta.

Encuesta: El evaluador encuesta primero la localización de todas las respuestas a la lámina y luego encuesta los determinantes, realiza preguntas directas (“¿por qué?”), induce las respuestas (“¿solo ves cosas por partes, no?").

Lámina favorita y lámina rechazada: Efectuada entre la fase de respuesta y la fase de encuesta, el evaluador olvida realizar esta elección y hace entrar nuevamente al examinado luego de haberlo despedido.

En cuanto a la autoevaluación que realiza el evaluador, este tiende a reconocer que la administración de la prueba despierta su ansiedad, pues le preocupa recordar y controlar todas sus instrucciones, a la par que mantener una actitud empática con el examinado y copiar todo lo dicho (algunos señalan que por copiar rápido, luego no entienden su letra y, por ello, en la encuesta no pueden repetir textualmente la respuesta al sujeto). Esta ansiedad se incrementa durante la fase de encuesta, debido a que en este momento el evaluador participa más activamente, buscando clarificar las respuestas del sujeto. Al respecto, al evaluador le resulta particularmente difícil elicitar los determinantes, sin llegar a inducirlos con sus preguntas, o a sobrentenderlos, dejando de encuestar. A la vez, como en este momento el diálogo es más fluido, les cuesta copiar todo textualmente. En contraste con la dificultad que les representa la encuesta, los evaluadores tienden a considerar que la fase de respuesta es más fácil de conducir.

En tanto, solo unos pocos evaluadores indican sentirse cómodos y tranquilos, pero es justamente a ellos a quienes les cuesta identificar sus errores en la administración, aspecto que se evidencia al contrastar la visualización del video con el informe que entregan.

Respecto al establecimiento del rapport, mientras un grupo de evaluadores lo considera un aspecto difícil, pues le cuesta identificar cuánta información 
es pertinente recabar sobre el examinado; otro grupo lo ve como un aspecto para el cual ya está entrenado, al ser una condición común a la aplicación de otras pruebas.

\section{DISCUSIÓN}

El psicodiagnóstico de Rorschach es un método que permite una evaluación completa y detallada, a la par que válida y fiable, de la organización y el funcionamiento de las personas, a partir de la estandarización de su aplicación, codificación e interpretación por el SCR. Se trata de un método exigente que requiere de una formación especializada dentro de los cursos dedicados a las pruebas psicológicas, de ahí que en las universidades más prestigiosas del país se dedique uno o dos ciclos enteramente a esta prueba, ubicándola además en los niveles superiores de la malla curricular y con otros cursos como prerrequisitos. En particular, el entrenamiento en su aplicación es crucial, pues de ello depende una correcta codificación y luego una adecuada interpretación. Este entrenamiento va incrementando gradualmente su complejidad conforme los estudiantes van incorporando todos los códigos del sistema. Así, no es sino hasta conocer todos los determinantes -características de la mancha que los examinados toman en cuenta para elaborar sus respuestas- que los estudiantes tienen la mayor parte de los elementos para desarrollar una encuesta apropiada.
El entrenamiento de la prueba es teórico y práctico, y el principal medio de verificación del aprendizaje del estudiante es el informe que este elabora. Sin embargo, este informe no suele reflejar todos los errores y dificultades que pueden haberse suscitado durante la aplicación, pues el estudiante puede no tener mucha claridad sobre ellos, lo que evidentemente dificulta corregirlos. Al respecto, una estrategia metodológica que ha resultado muy efectiva para la autora en su calidad de docente es la filmación de la aplicación realizada por los estudiantes; y recientemente, acompañando esta filmación, la elaboración de la ficha de observación sobre la que se realiza este estudio. Esta ficha de observación permite a los estudiantes estar más conscientes de las numerosas instrucciones que deben seguir (lo que se ve favorecido por el trabajo en pares con roles intercalados de evaluador y observador), a la vez, que permite a los docentes monitorear más efectivamente su manejo en la administración de la prueba.

Por otro lado, así como el examinado pasa por un complejo proceso perceptivo-cognitivo para elaborar su respuesta, de igual forma el evaluador enfrenta una tarea exigente al administrar la prueba, pues sus varias instrucciones y la simultaneidad de las operaciones implicadas en ella demandan no solo un conocimiento sólido del Rorschach, sino también una serie de destrezas cognitivas e interpersonales que 
complementan su manejo técnico. Estas destrezas cognitivas e interpersonales son críticas para obtener un protocolo válido.

Uno de los principales hallazgos de este estudio tiene que ver precisamente con la capacidad de los estudiantes para generar un ambiente cómodo y cálido, basado -como señala Lerner (1996a, 1996b) - en la empatía; la misma que demanda una atención especial en el caso de los examinados con muy pocas experiencias de evaluación, pues ellos carecerían de esquemas que les permitan adaptarse a esta situación (Acklin, 1994), como ocurre de modo natural con los niños. Similar atención se requiere cuando el examinado y el evaluador son marcadamente distintos (Vinet, 2000), lo que tiene particular relevancia para un país multiétnico, pluricultural, multilingüe y multiconfesional como el nuestro, donde las diferencias entre unos y otros pueden marcar enormes brechas. Así, puede suceder que la administración del Rorschach sea tanto una experiencia nueva como ajena al panorama cultural del examinado (Daroglou, 2009).

En este estudio la disposición de un ambiente luminoso y libre de interrupciones y ruidos es provisto por la universidad. Sin embargo, la comodidad y calidez, que dependen del establecimiento de un rapport adecuado, presentan serias limitaciones. Si bien la complejidad de la aplicación del Rorschach puede generar inseguridad y ansiedad en el evaluador, es significativa su falta de atención al examinado, dedicándole poco o ningún tiempo para conocerlo (no preguntan datos básicos ni antecedentes) y para que este se familiarice con el evaluador y la situación de evaluación (no explican el procedimiento).

Al respecto, el establecimiento del vínculo con el examinado no es una competencia nueva para estos estudiantes, quienes se encuentran en un nivel avanzado de la carrera y ya han llevado varios cursos en los que se aborda y trabaja la importancia del rapport. Por tanto, aunque es una dificultad que se evidencia con la aplicación del Rorschach, va más allá de esta prueba, mostrando el logro desigual de esta habilidad en el conjunto de los estudiantes. Así, mientras para algunos -los menos- el establecimiento del rapport es un aspecto sencillo al haberlo incorporado en varios cursos previos; para otros -los más- resulta difícil, generando la impresión de estar desprovistos de un esquema en el rol de evaluadores al costarles manejar la interacción con el examinado. Lo anterior nos plantea que si bien algunos estudiantes desarrollan esta competencia bajo el sistema de enseñanza que se les brinda, para que esta se asiente la mayoría parece requerir que se intensifiquen la supervisión, el seguimiento y el modelamiento de su interacción con los examinados.

La supervisión en este estudio implica un doble rol para el evaluador: a la vez que evalúa a un sujeto, él mismo 
es evaluado en su performance, tanto por el par que registra su desempeño como por los responsables de la cátedra. Es probable que para muchos sea la primera vez que se vean entrenados de esta manera y les cueste mostrarse a los demás. A la vez, mientras realizan la aplicación pueden estar muy conscientes sobre los aspectos en los que están fallando debido a que teóricamente conocen las dificultades que pueden presentarse, pero todavía no han desarrollado las respuestas para enfrentarlas. Todo ello parece incrementar el nivel de ansiedad e incomodidad del evaluador, lo que se evidencia en el apuro por concluir la evaluación (la cual termina rápidamente para la gran mayoría) y en la relación distante que se genera con el examinado.

Como señala Exner (1994), lo anterior puede afectar el desempeño de los examinados. En este estudio, en contraste con los evaluadores, la mayor parte de ellos luce calmada, aunque un porcentaje importante también muestra signos de apuro o ansiedad, lo que como es evidente no contribuye a la elaboración de sus respuestas a la prueba. Asimismo, los examinados tienden a interrumpir o a salirse del proceso de la fase de respuesta, haciendo preguntas al evaluador. Esta conducta no parece ser provocada por la ansiedad o una actitud defensiva (la gran mayoría toma las láminas en sus manos, brinda más de una respuesta a la primera lámina y ninguno se bloquea), sino básicamente por el desconcierto que les produce la situación de evaluación y de la misma prueba (recordemos que se trata de una prueba compuesta por estímulos inestructurados y ambigüos). De esta manera, las dificultades para el establecimiento del rapport y el estado del evaluador tienen un impacto directo sobre la evaluación, por lo que aun cuando la prueba sea válida y confiable, bajo estas condiciones la información que recaba es pobre y puede no representar en forma adecuada al examinado.

Consideramos además que la falta de rapport y empatía, así como las variables que interfieren en el desempeño del evaluador, pueden alterar los resultados de cualquier prueba psicológica en la que la interacción humana entre en juego, no solo la del Rorschach. Al respecto, si como plantean Dana (2009) y Daroglou (2009) el entrenamiento de los evaluadores sigue dependiendo de aspectos procedimentales disímiles culturalmente de ellos, la incorporación de la perspectiva cultural implicaría entender mejor cómo construimos relaciones en nuestra sociedad, para lo cual es necesario tener presente nuestra idiosincrasia, valores, costumbres, experiencias, entre otros. En la experiencia de la autora cuando aplicaba el Rorschach a niños cuya lengua materna es el quechua, ha notado que al ser presentada por alguien que cumple un rol valorado en la comunidad, al saludarlos y preguntarles sus nombres en quechua, y mostrar algún conocimiento sobre el 
lugar donde viven y las actividades que realizan, cambia notablemente la calidad de la interacción, tornándola más cercana y confiable.

Sin embargo, incluso para los evaluadores más empáticos, el número total de instrucciones para la aplicación del Rorschach puede resultar abrumador cuando se empieza a conocer la prueba. De ahí que resulte más sencillo cumplir con las instrucciones más automáticas (copiar todo literalmente en la fase de respuesta, encuestar cada respuesta de manera individualizada, entre otras) que con aquellas que demanden su adaptación (la explicación de la consigna de la encuesta) o la intervención consciente del evaluador (la manipulación de láminas). Lo anterior es particularmente cierto en la fase de encuesta, donde los evaluadores presentan varias dificultades, no tanto porque el número de instrucciones se incremente significativamente, sino porque esta implica una interacción constante y activa con el examinado a la par que se operan en forma simultánea otras tareas cognitivas. Paulatinamente, el entrenamiento técnico de la prueba permitirá a los estudiantes saber qué preguntas específicas puede hacer en la encuesta para elicitar los determinantes, pero si no se pone igual énfasis en el desarrollo de su capacidad para interactuar con el examinado es probable que varios de los errores detectados persistan.

Por otro lado, considerando la variable género, encontramos algunas ten- dencias que resultan llamativas. Entre los varones existe una mayor dificultad para acomodar los muebles del ambiente buscando la comodidad del examinado y la suya propia, organizar las láminas por el reverso, entregar la primera lámina en las manos del examinado, repetir textualmente la respuesta del sujeto y encuestar siempre todos los determinantes. Además, suelen provocar una actitud más apurada en los examinados. En tanto, entre las mujeres surgen más problemas para ubicarse en una posición más cómoda respecto al examinado, consultarle sus datos de filiación $\mathrm{y}$ antecedentes, solicitarle que brinde más respuestas a la primera lámina y encuestar la localización. ${ }^{2}$ Asimismo, ellas suelen generar una respuesta más ansiosa en los examinados. Al respecto, mientras en los evaluadores hombres observamos una cierta desidia y apuro, en las evaluadoras mujeres apreciamos un nivel de ansiedad que perturba el intercambio con el examinado, costándoles asumir una posición más asertiva con ellos.

Finalmente, como hemos revisado, el psicodiagnóstico de Rorschach implica un método complejo en el que interactúan múltiples factores para determinar su aplicación adecuada: el ambiente, el examinado, el conocimiento, la expe-

$\overline{2 \mathrm{Al} \text { respecto, }}$ varias evaluadoras indican que omiten esta pregunta porque el examinado espontáneamente muestra la localización de la respuesta, lo que para ellas resulta claro. 
riencia y la destreza del evaluador, y la dinámica interpersonal que se suscita entre examinado y evaluador. En este estudio hemos buscado monitorear los factores mencionados mediante la elaboración de una ficha de observación que recoja todas las instrucciones requeridas para la aplicación de la prueba. Este instrumento ha sido integrado exitosamente en la metodología de la clase, lo que ha permitido, en la experiencia de la autora, estimar cualitativamente el avance del alumno en su proceso de aprendizaje. Lo anterior permite tomar decisiones pedagógicas a fin de ajustar y mejorar este proceso, buscando favorecer el desarrollo de destrezas críticas en los estudiantes para un desempeño adecuado y eficiente en la aplicación de pruebas como el Rorschach.

\section{REFERENCIAS}

Acklin, M. W. (1994). Some contributions of cognitive science to the Rorschach Test. En I. B. Weiner (Ed.). Rorschachiana XIX (pp. 129145). Bern, Switzerland: Hogrefe and Huber.

Allen, J., \& Dana, R. H. (2004). Methodological issues in cross-cultural and multicultural Rorschach research. Journal of Personality Assessment, 82, 189-206.

Dana, R. H. (2009). Rorschach and the Rorschach comprehensive system. En R. H. Dana (Ed.). Multicultural assessment. Principles, applications, and ex- amples. Nueva Jersey: Lawrence Erlbaum Associates, pp. 121-145.

Daroglou, S. (2009). Greek assessees (Rorschach CS). En Dana, R. (Ed.). Multicultural assessment. Principles, applications, and examples. Nueva York: Psychology Press.

Ephraim, D. (1996). El método Rorschach en la actualidad. Caracas: Facultad de Humanidades y Educación de la Universidad Central de Venezuela / Monte Ávila Editores Latinoamericana.

Exner, J. E. (2005). Principios de interpretación del Rorschach. Un manual para el sistema comprehensivo. Madrid: Psimática.

Exner, J. (2003). Manual de codificación del Rorschach para el sistema comprehensivo. (5. ${ }^{\mathrm{a}}$ edición). Madrid: Psimática.

Exner, J. E. (1994). El Rorschach. Un sistema comprehensivo. Vol. 1: Fundamentos Básicos. Madrid: Psimática.

Exner, J. E. \& Weiner, I. (1995). The Rorschach: A comprehensive system. Vol. 3: Assessment of children and adolescents. Nueva York: John Wiley \& Sons, Inc.

Franchi, V. \& Andronikof-Sanglade (1999). Methodological and epistemological issues raised by the use of the Rorschach comprehensive system in cross-cultural research. En Andronikof-Sanglade, A. (Ed.). 
Rorschachiana XXIII Yearbook of the International Rorschach Society (pp. 118-134). Göttingen: Hogrefe and Huber Publishers.

Lerner, P. M. (1996a). Current perspectives on psychoanalytic Rorschach assessment. Journal of Personality Assessment, 67(3), 450-461.

Lerner, P. M. (1996b). The interpretive process in Rorschach testing. Journal of Personality Assessment, 67(3), 494-500.

Lis, A.; Parolin, L.; Calvo, V.; Zennaro, A. \& Meyer, G. (2007). The impact of administration and inquiry on Rorschach comprehensive system protocols in a national reference sample. Journal of Personality Assesment, 89 (S1), 193-200.

Ráez, M. (2-5 de agosto, 2006). "Enseñanza de las pruebas psicológicas en el Perú. Perspectivas futuras". Ponencia presentada en el XIII Congreso Latinoamericano de Rorschach y otras Técnicas Proyectivas.

Vinet, E. (2000). The Rorschach comprehensive system in Iberoamerica. En Dana, R. (Ed.). Handbook of cross-cultural and multicultural personality assessment. (pp. 345365). New Jersey: Lawrence Erlbaum Associates.

Weiner, I. (1997). Current status of the Rorschach inkblot method. Journal of Personality, 68, 5-19. 